\title{
ON LIKELIHOOD RATIOS OF MEASURES GIVEN BY MARKOV CHAINS
}

\author{
R. LePAGE AND V. MANDREKAR ${ }^{1}$
}

ABSTRACT. In this note we study conditions for absolute continuity and singularity of two measures induced by finite Markov chains. These conditions are derived from a general result on singularity-continuity dichotomy.

1. In [3] we considered, for general measures, the relation between equivalence-singularity dichotomies and zero-one laws with respect to the tail field of transition densities. In [4] Lodkin studied, under the exact analogue of Kolmogorov's zero-one law, the problem of the existence of the densities for two Markov chain measures on the space of real sequences. Unfortunately, the proof of Lodkin [4, Theorem 2], on which this theorem is based, is incomplete. In addition, Lodkin's conditions for the Markov case [4, Theorem 3] fail to cover the special case of the products of discrete measures, which was completely solved by Kakutani [1].

We first state a slight generalization of our dichotomy result for probability measures [3], which includes Lodkin [4, Theorem 2]. From this, sufficient conditions are derived for absolute continuity of one Markov chain measure with respect to another. These conditions improve those of Lodkin [4, Theorem 3]. Specializing further, we obtain conditions necessary and sufficient for absolute continuity of one Markov chain measure with respect to another, when a particular Condition $A$ is satisfied. A large class of pairs of Markov chain measures is shown to satisfy Condition A. In particular, pairs of discrete product measures satisfy Condition $A$, and for this case the n.a.s. conditions we obtain for absolute continuity are exact analogues of those obtained by Kakutani [1, Corollary 1] in the Bernoulli case.

1.1. Theorem. Let $\mathcal{F}_{1} \subset \mathcal{F}_{2} \subset \cdots$ be $\sigma$-algebras of subsets of $\Omega, \mathcal{F}=$ $\sigma\left(\bigcup_{n} \mathcal{F}_{n}\right)$ the $\sigma$-algebra generated by their union. For any probability measures $P, Q$ on $\mathcal{F}$ with $Q$ absolutely continuous with respect to $P(Q \ll P)$ when restricted to each of $\mathcal{F}_{1}, \mathcal{F}_{2}, \ldots$, we let $\rho_{n}=\left(d Q \mid \mathcal{F}_{n}\right) /\left(d P \mid \mathcal{F}_{n}\right)$ and define the tail $\sigma$-algebra $\mathcal{G}=\bigcap_{n} \sigma\left\{\rho_{k+1} / \rho_{k} \mid k \geq n\right\}$. The following is then true: $(A \in \mathcal{G} \Rightarrow Q(A)=0$ or 1 i.e. $\mathcal{G}$ is $Q$ trivial $) \Rightarrow P \perp Q$ or $Q \ll P$ on

Received by the editors January 31, 1974 and, in revised form, June 14, 1974 and August 9, 1974.

AMS (MOS) subject classifications (1970). Primary 60G30; Secondary 60F 20.

Key words and phrases. Singularity, absolute continuity, measures induced by Markov chains, zero-one law .

1 Partially supported by contract NSF GP-28658X.

Copyright $\odot$ 1975, American Mathematical Society. 
F. Furthermore, if $\mathcal{G}$ is $Q$-trivial, we have $Q \ll P$ on $\mathcal{F}$ iff $E_{P} \rho_{n}^{1 / 2} \nrightarrow 0$.

The first part of this theorem is proved as is the theorem in [3]. The second part is readily derived from the work of C. Kraft [2].

1.2. Remark. The error in [4] occurs since the author asserts [4, p. 692] that if $P, Q$ are two measures such that $\partial Q / \partial P$ (the Radon-Nykodym derivative of the absolutely continuous part of $Q$ with respect to $P$ ) satisfies $Q(\partial Q / \partial P>0)=1$, then $Q \ll P$. We caution that the Radon-Nykodym derivative $\partial Q / \partial P$ is defined a.e. $P$. Consider now $\Omega=\{0,1\}, \mathcal{F}=$ the $\sigma$.field all subsets of $\Omega$. Define $Q\{i\}=1 / 2(i=0,1)$ and $P\{0\}=1, P\{1\}=0$. Then $f$ defined by $f(0)=1 / 2, f(1)=+\infty$ is a version of $\partial Q / \partial P$ (in some respects the most natural), and $Q(f>0)=1$. But $Q$ is not absolutely continuous with respect to $P$. This difficulty was specifically avoided in [3].

Now, in view of Theorem 1.1, it suffices to obtain conditions for $E_{P} \rho_{n}^{1 / 2}$ t 0 once we assume that our zero-one law applies with respect to $Q$. Throughout the remainder we shall assume $\varrho$ is $Q$-trivial.

2. Let $\Omega_{k}=\{1,2, \ldots, N\}, \Omega=\Pi_{k} \Omega_{k}$ and $P, Q$ be two Markov measures given by the respective starting vectors $\left\{p_{0}(i)\right\}_{i=1}^{N},\left\{q_{0}(i)\right\}_{i=1}^{N}$, and matrices of transitions $\left\{p_{n}(i, j)\right\}_{i, j=1}^{N},\left\{q_{n}(i, j)\right\}_{i, j=1}^{N}, n=1,2, \ldots$ Let $P_{n}$ and $Q_{n}$ $(n=1,2, \ldots)$ be the corresponding Markov measures on the $\sigma$-algebra $\mathcal{F}_{n}$ of cylinder sets with base a subset of $\Pi_{k=1}^{n} \Omega_{k}$. Suppose $Q_{n} \ll P_{n}$ and $\rho_{n}=$ $d Q_{n} / d P_{n}, \forall n \geq 1$. In general, we know that

$$
E_{P} \rho_{n}^{1 / 2} \nrightarrow 0 \text { iff } \prod_{n} \frac{E_{P} \rho_{n+1}^{1 / 2}}{E_{P} \rho_{n}^{1 / 2}} \text { converges iff } \sum_{n}\left(1-\frac{E_{P} \rho_{n+1}^{1 / 2}}{E_{P} \rho_{n}^{1 / 2}}\right)<\infty .
$$

Hence under our zero-one law the condition $\Sigma_{n}\left(1-E_{P} \rho_{n+1}^{1 / 2} / E_{P} \rho_{n}^{1 / 2}\right)<\infty$ is necessary and sufficient for the absolute continuity of $Q$ with respect to $P$ on $\mathfrak{F}=\sigma\left(\bigcup_{n} \mathcal{F}_{n}\right)$.

For Markov measures we note that [4, p. 693] letting

$$
a_{x_{n}}^{n}=\sum_{x_{1} \cdots x_{n-1}} \sqrt{p_{0}\left(x_{1}\right) q_{0}\left(x_{1}\right) \cdots p_{n-1}\left(x_{n-1}, x_{n}\right) q_{n-1}\left(x_{n-1}, x_{n}\right)}
$$

and $I_{n}=E_{P} \rho_{n}^{1 / 2}$, we have

$$
\frac{I_{n+1}}{I_{n}}=\sum_{x_{n}} a_{x_{n}}^{n}\left\{\sum_{j=1}^{N} \sqrt{p_{n}^{\left(x_{n, j}\right)}} \sqrt{q_{n}\left(x_{n, j}\right)}\right\} / \sum_{x_{n}} a_{x_{n}}^{n} .
$$

Hence

$$
\left(1-\frac{I_{n+1}}{I_{n}}\right)=\sum_{i} \frac{a_{i}^{n}}{\sum_{k} a_{k}^{n}} \sum_{j=1}^{N}\left(\sqrt{p_{n}^{(i, j)}}-\sqrt{q_{n}^{(i, j)}}\right)^{2} .
$$


From equation (2.3) the following proposition is obvious.

\subsection{Proposition.}

$\sum_{n}\left\{\sum_{j=1}^{N}\left(\sqrt{p_{n}^{(i, j)}}-\sqrt{\left.q_{n}^{(i, j)}\right)^{2}}\right\}<\infty \forall i \Rightarrow \sum_{n}\left(1-\frac{E_{P} \rho_{n+1}^{1 / 2}}{E_{P} \rho_{n}^{1 / 2}}\right)<\infty\right.$.

Since $\left(\sqrt{p_{n}(i, j)}-\sqrt{q_{n}(i, j)}\right)^{2} \leq\left(p_{n}(i, j)-q_{n}(i, j)\right)^{2} / p_{n}(i, j)$ for all $i, j$, the above proposition gives Theorem 3 of [4].

We now specialize to a class of pairs of Markov chain measures for which the conditions above take simpler form.

Condition A. There exists $\delta>0$,

$$
E_{p}^{x} n-1 \sqrt{q_{n-1}\left(x_{n-1}, x_{n}\right) / p_{n-1}\left(x_{n-1}, x_{n}\right)} g_{n}\left(x_{n}\right) \geq \delta \sum_{i=1}^{N} g_{n}(i)
$$

for sufficiently large $n$ a.e. $[P]$, where $g_{n}\left(x_{n}\right)=\Sigma_{j}\left(\sqrt{p_{n}\left(x_{n}, j\right)}-\sqrt{q_{n}\left(x_{n}, j\right)}\right)^{2}$.

The following theorem gives necessary and sufficient conditions for existence of densities subject to $A$.

2.5. Theorem. Let $P$ and $Q$ be Markov chain measures on $\Omega$ such that (i) $Q_{n} \ll P_{n}$, (ii) $\mathcal{Y}$ is Q-trivial, and (iii) Condition $\mathrm{A}$ is satisfied. Then $\Sigma_{n}^{\infty}\left(\sqrt{p_{n}(i, j)}-\sqrt{q_{n}(i, j)}\right)^{2}<\infty$ for all $i, j=1,2, \ldots, N$ iff $Q \ll P$ on $\mathcal{F}$, otherwise $Q \perp P$ on $\mathcal{F}$.

Proof. In view of Proposition 2.4 it suffices to prove that $Q \ll P$ implies $\Sigma_{n}\left(\sqrt{p_{n}(i, j)}-\sqrt{q_{n}(i, j)}\right)^{2}<\infty$ for all $i, j=1,2, \ldots, N$. We first note the numerator of the right side of (2.3) can be written as

$$
E P \sqrt{\frac{q_{0}\left(x_{1}\right)}{p_{0}\left(x_{1}\right)}} E^{x_{1}} \sqrt{\frac{q_{1}\left(x_{1}, x_{2}\right)}{p_{1}\left(x_{1}, x_{2}\right)}} \cdots E^{x} n-1 \sqrt{\frac{q_{n-1}\left(x_{n-1}, x_{n}\right)}{p_{n-1}\left(x_{n-1}, x_{n}\right)}} g_{n}\left(x_{n}\right)
$$

where $E^{x_{i}}$ denotes the conditional expectation given $x_{i}$, and $g_{n}\left(x_{n}\right)=$ $\Sigma_{j}\left(\sqrt{p_{n}\left(x_{n}, j\right)}-\sqrt{q_{n}\left(x_{n}, j\right)}\right)^{2}$. From (2.6) and Condition A we get

$$
\left(1-\frac{I_{n+1}}{I_{n}}\right) \geq \delta \frac{I_{n-1}}{I_{n}} \sum_{i=1}^{N} g_{n}(i)
$$

If the sum on the right side of (2.1) is finite (i.e. if $Q \ll P$ ), then there is an $M$ such that for $n \geq M$ we get $I_{n-1} / I_{n}>1 / 2$. From the above we have that for $n \geq M,\left(1-I_{n+1} / I_{n}\right) \geq 1 / 2 \delta \sum_{i=1}^{N} g_{n}(i)$. This implies the result.

2.7. Remark. (i) The above proof also shows that if $P$ and $Q$ obey Condition $A$ and $Q \ll P$, then $\Sigma_{n}\left(\sqrt{p_{n}(i, j)}-\sqrt{q_{n}(i, j)}\right)^{2}<\infty$ for all $i, j$. (ii) If $P$ and $Q$ are Markov chain measures on $\Omega$ with $Q$-trivial and if $\left\{p_{0}(i)\right\}_{i=1}^{N},\left\{q_{0}(i)\right\}_{i=1}^{N},\left\{p_{n}(i, j)\right\}_{i, j=1}^{N},\left\{q_{n}(i, j)\right\}_{i, j=1}^{N}(n=1,2, \ldots)$ are bounded 
away from zero then

$$
E^{x} n-1 \sqrt{\frac{q_{n}\left(x_{n-1}, x_{n}\right)}{p_{n}\left(x_{n-1}, x_{n}\right)}} g_{n}\left(x_{n}\right) \geq \sqrt{\delta} \sum_{i=1}^{N} \sqrt{p_{n-1}\left(x_{n-1}, i\right)} g_{n}(i) \geq \delta \sum_{i=1}^{N} g_{n}(i) .
$$

Hence Condition A is satisfied for measures in this class, and Theorem 2.5 therefore furnishes necessary and sufficient conditions for $Q \ll P$ on $\mathfrak{F}$.

We now derive the results of Kakutani where the measures $P$ and $Q$ on $\Omega$ are product measures. We note that in this case $p_{n}(i, j)=p_{n}(j)$ independent of $i$. Also $I_{n+1} / I_{n}=\sum_{j=1}^{N} \sqrt{p_{n}(j)} \sqrt{q_{n}(j)}$ and

$$
E^{x} n-1 \sqrt{\frac{q_{n-1}\left(x_{n-1}, x_{n}\right)}{p_{n}\left(x_{n-1}, x_{n}\right)}} g_{n}\left(x_{n}\right)=E P \sqrt{\frac{q_{n-1}\left(x_{n}\right)}{p_{n-1}\left(x_{n}\right)}} g_{n}\left(x_{n}\right) \text {. }
$$

We note that $g_{n}\left(x_{n}\right)=\sum_{j=1}^{N}\left(\sqrt{p_{n}(j)}-\sqrt{q_{n}(j)}\right)^{2}$ is independent of $x_{n}$, and (2.8) is therefore equal to $\sum_{i=1}^{N} \sqrt{q_{n-1}(i)} \sqrt{p_{n-1}(i)} \sum_{j=1}^{N}\left(\sqrt{p_{n}^{(j)}}-\sqrt{q_{n}(j)}\right)^{2}$. If $Q \ll$ $P$ then from (2.1), for $\delta>0, \exists N_{\delta}$ such that $\Sigma_{i=1}^{N} \sqrt{q_{n-1}(i)} \sqrt{p_{n-1}(i)}>\delta$ for $n \geq N_{\delta}$. Hence $Q \ll P$ implies that $P$ and $Q$ satisfy Condition A. From Proposition 2.4 and Remark 2.7(i) we get the following which includes Kakutani [1, Corollary 1] since for product measures $\mathcal{G}$ is always $Q$-trivial [3].

2.9. Corollary. If $P$ and $Q$ are product measures on $\Omega$ as above such that $Q_{n} \ll P_{n^{\prime}}$ then $Q \ll P$ on $\mathcal{F}$ iff $\Sigma_{n}\left(\sqrt{p_{n}(i)}-\sqrt{q_{n}(i)}\right)^{2}<\infty$ for $i=1$, $2, \ldots, N$.

\section{REFERENCES}

1. S. Kaķutani, On equivalence of infinite product measures, Ann. of Math. (2) 49 (1948), 214-224. MR 9, 340.

2. C. Kraft, Some conditions for consistency and uniform consistency of statistical procedures, Univ. California Publ. Statist. 2 (1955), 125-141. MR 17, 505.

3. R. LePage and V. Mandrekar, Equivalence-singularity dichotomies from zero-one laws, Proc. Amer. Math. Soc. 31 (1972), 251-254. MR 44 \#7623.

4. A. A. Lodkin, The absolute continuity of measures that correspond to Markov processes with discrete time, Teor. Verojatnost. i Primenen. 16 (1971), 703708 = Theor. Probability Appl. 16 (1971), 690-694. MR $45 \# 497$.

DEPARTMENT OF STATISTICS AND PROBABILITY, MICHIGAN STATE UNIVERSITY, EAST LANSING, MICHIGAN 48823 